\title{
Clinical study on the sensitivity test guided hepato-arterial/portal-vein chemotherapy in patients with unresectable hepatocellar carcinoma
}

\author{
Dongde $\mathrm{Wu}^{1}$, Shaozhong $\mathrm{Wei}^{2}{ }^{*}$, Chenggang $\mathrm{Luo}^{1}$, Xinghong $\mathrm{Wu}^{3}$, Yaojun Feng ${ }^{3}$, \\ Feng Zhang ${ }^{1}$, Lei Nie ${ }^{1}$, Xiaoqin $\mathrm{Xia}^{1}$ \\ ${ }^{I}$ Department of Hepatobiliary and Pancreatic Surgery, Cancer Hospital of Wuhan University, Wuhan, Hubei, China. \\ ${ }^{2}$ Department of Urology Surgery, Cancer Hospital of Wuhan University, Wuhan, Hubei, China. \\ ${ }^{3}$ Department of Breast Cancer Surgery, Cancer Hospital of Wuhan University, Wuhan, Hubei, China.
}

Received February 11, 2014; Revised April 04, 2014; Accepted April 06, 2014; Published Online April 12, 2014

\section{Original Article}

\begin{abstract}
Purpose: Approximately $20 \%$ of tumors have the opportunity to be resected in patients with hepatocellar carcinoma (HCC) and their prognoses were acceptable. For the unrespectable HCC, however, the outcomes were rather poor because the specialty of the tumor blood supply and the tumor was insensitive to the chemotherapy drug. The objective of this study was to find sensitive drugs for individual patients and determine the safety and antitumor activity of hepatic intra-arterial and portal vein infusion chemotherapy. Methods: A total of 120 patients with the mean patient age of 56 years and with unresectable HCC were randomly divided into experimental group and control group. The experiment group was infused through an intra-arterial and portal vein catheter 3 different drugs were chosen by the results of drug sensitive test, whereas the control group was treated for TACE. The changes in tumor-size and AFP, two-step operation rate, survival rate, and complications were observed in these patients. Results: The tumor size reduced in $28(47.6 \%)$ cases, stabilized in $14(23.3 \%)$ cases, and progressed in $18(30 \%)$ cases in experimental group as measured by CT or MRI after six chemotherapy cycles, whereas the corresponding data was 17 (28.5\%) cases, $7(11.5 \%)$ cases and $36(60 \%)$ cases in the control group $(\mathrm{P}<0.05)$. The AFP was declined in 51 cases in experimental group and in 30 cases in control group $(\mathrm{P}<0.05)$. In the experimental group, the median follow-up time was 21 months; the overall survival rates (OS) of 6 months, 12 months, and 18 months were $86 \%, 72 \%$, and $65 \%$, respectively. In the control group, the median follow-up time was 16 months; the OS rates of 6 months and 12 months were $58 \%$ and $40 \%$, respectively. Six patients in the experimental group and 3 patients in the control group had two-step operation. There was no severe incidence of complications in both groups. In the experimental group, 2 (3\%) patients had wound infection, 8 cases had the chemotherapy relative diarrhea, and $18(30 \%)$ cases had grade I or II bone marrow suppression. In the control group, the chemotherapy relative diarrheas were $15(25 \%)$ cases and grade I or II bone marrow suppression were $33(55 \%)$ cases. Conclusion: The artery and portal vein pump transfusion chemotherapy guided by drug sensitive test was efficient for HCC treatment. The patients can get longer OS and lower complication incidence.
\end{abstract}

Keywords: Primary Liver Cancer; Drug Sensitive Test; Regional Chemotherapy

\section{Introduction}

Because the liver tumors are not easily found, only less than $20 \%$ of the primary liver cancers have the opportunity to be

Corresponding author: Shaozhong Wei; Cancer Hospital of Wuhan University, Zhuodaoquan South Road, Hongshan district, Wuhan, 430079, China.

Cite this article as:

Wu D, Wei S, Luo C, Wu X, Feng Y, Zhang F, Nie L, Xia X. Clinical study on the sensitivity test guided hepato-arterial/portal -vein chemotherapy in patients with unresectable hepatocellar carcinoma. Int J Cancer Ther Oncol 2014; 2(2):02029.

DOI: $10.14319 /$ ijcto.0202.9 surgically removed. Treatment of hepatic artery chemotherapy embolism (TACE) is one of the therapeutics available to cure the advanced stage unresectable liver cancer; however, the effective rate is about $30 \%$ to $40 \%$. Research showed that primary liver cancers have hepatic artery and portal vein dual blood supplies: blood vessel in the center of the tumor is mainly composed of hepatic artery whereas the peripheral part is mainly composed of portal vein. The cancer cells tend to invade the intrahepatic vascular, especially into the portal system, which promote the portal venous embolism and 
have negative effects of conventional TACE. In order to improve the efficiency to cure primary liver cancer, scholars developed the hepatic artery/portal vein pump chemotherapy (HA/PVPC). ${ }^{1}$ However, liver cancer is not aeschynomenous to chemotherapy. To improve the efficiency of individual patiens to HA/PVPC, we used individualized drug to patients based on their sensitive tests (MTT) and perfused the drugs through transartery and portal vein dual channel. We aimed to increase the regional drug concentration and improve the tumor susceptibility to drugs, thus improving the efficiency of liver cancer treatment.

\section{Methods and Materials}

\section{Patients}

Between October, 2010 and October, 2005, 120 cases of unresectable HCC were randomly divided into experimental group and control group. 60 patients in the experimental group were enrolled to determine the safety and antitumor activity of hepatic intra-arterial and portal vein infusion chemotherapy; another 60 patients were enrolled in the control group. The clinical protocol was reviewed and approved by the Institutional Review Board of the Hubei Cancer Hospital. All study participants provided written informed consent prior to therapy. Eligible study participants were chosen according to the following inclusion criteria:

- $\quad$ Liver function test was Child A-B grade;

- Tumor cannot be resected;

- $\quad$ Eastern Cooperative Oncology Group performance status was 0 or 1 ;

- Total bilirubin concentration was $\leq 1.0 \mathrm{mg} / \mathrm{dL}$;

- Serum creatinine concentration was $\leq 1.5 \mathrm{mg} / \mathrm{dL}$;

- Prothrombin time was $<13$ seconds; activated partial thromboplastin time was $<30$ seconds; and

- Complete blood count was normal. All of the patients before treatment did not use any antitumor drugs.

\section{Preparation of tumor cells}

Sampling: Specimens of primary liver cancer tissue was obtained from surgical biopsy, placed into RPMI 1640 culture medium which contained streptomycin $(100 \mathrm{U} / \mathrm{ml})$ and penicillin streptomycin $(100 \mathrm{mg} / \mathrm{ml})$ within 10 minutes after shearing, preserved in $4{ }^{\circ} \mathrm{C}$ refrigerator, and processed as described below within 4 hours.

Processing samples: Tissue blocks were placed in a petridish, cut off blood clots and unused tissues, rinsed with hanks solution twice, and then cut into $1 \mathrm{~mm}^{3}$ tissue blocks.

Isolating cells: We crushed the small tissue blocks gently by repeatly passing through a syringe needle, The tissue homogenate was filtered through 4 layers of gauze followed by centrifugation in low speed centrifuge machine $(800 \mathrm{rpm}, 5$ min) twice.

\section{Cell culture}

Cells were cultured in RPMI 1640 (Gibco, Invitrogen) supplemented with $10 \%(\mathrm{v} / \mathrm{v})$ heat inactivated fetal bovine serum (Gibco, Invitrogen) and 1\% (v/v) antimicotic antibiotic (Gibco, Invitrogen)at $37^{\circ} \mathrm{C}$ under a $5 \% \mathrm{CO}_{2}$ atmosphere. The cells adhered to the wall of the culture flasks after 48 hours. After 4 days of incubation, the cell concentration was adjusted to (2 to 5$) \times 10^{5} / \mathrm{ml}$.

\section{Cell viability assay (MTT assay)}

100 ul of liver cancer cells were seeded on 96-well plates. For the cells in the experimental group, seven chemotherapy drugs (5-FU/ DDP/ ADM/ MMC/ HCPT/ LOHP/ GEM) were individually added to the cells in triplicate at a concentration ten times higher than that used clinically. No drug was added to the cells in control group. 1640 medium was used as a blank control. Cells were cultured for 48 hours. Then the cells were added $20 \mathrm{ml}$ of MTT $(5 \mathrm{mg} / \mathrm{ml})$ and continually cultured for 4 hours before replacing culture medium with dimethyl sulfoxide (100 $\mu \mathrm{L} /$ well $)$ to terminate the reaction. The cells were finally vibrated for 5 minutes and the absorbance (value A) was measured by a micro plate reader at a wavelength of $570 \mathrm{~nm}$.

Drugs inhibition rate $=[1-$ average $\mathrm{A}$ value in drug treatment wells / average A value in negative control wells)]. A rate value $>50 \%$ is defined as hypersensitivity; $30 \%$ to $50 \%$ as mild sensitive; $<30 \%$ as drug resistance.

Sensitive rate $(\%)=($ sensitive cases $/$ total cases of the group $)$ $\times 100 \%$

\section{Clinical experiment}

All patients in the experimental group underwent angiographic placement of two catheters for hepato-artory and portal vein. We obtained the hepatic tumor biopsy for pathological diagnosis and then The Bard Access Ports (detailed information showed below) were inserted into the right gastro-omental artery and the right gastro-omental vein to establish regional chemotherapy channels. Seven chemotherapy drugs were used as the experimental drugs (detailed information showed below). We infused each individual in the experimental group by arterial and portal vein pump 2 to 3 drugs which showed highest inhibition to the cancers of the patient in our MTT test above. The drugs were delivered equally in both channels. The regimen was repeated every 3 weeks unless there was a tumor progression, withdrawal of consent, or occurrence of side effects, such as bone marrow suppression, fulminant hepatitis, or other greater than grade 3 toxicities, etc.

The control group patients underwent conventional TACE, chemotherapy regimens were 5 - fluorouracil $\left(1000 \mathrm{mg} / \mathrm{m}^{2}\right)$, 
mitomycin $\left(10 \mathrm{mg} / \mathrm{m}^{2}\right)$ and cisplatin $\left(100 \mathrm{mg} / \mathrm{m}^{2}\right)$, and with iodipin embolization (scheme: $\mathrm{MMC}+\mathrm{DDP}+5-\mathrm{Fu}$, iodipin embolism).

The Bard Access Ports was purchased from West Amelia Earhart Drive, Salt Lake City, Utah, USA. The cytotoxic drugs included fluorouracil (5 FU, Tianjin Jinyao acid co., LTD), cisplatin (DDP, Yunnan Gejiu Supertrack Bio-Pharmaceutical co.,LTD), adriamycin (ADM, Zhejiang Hisun pharmaceutical co., LTD), mitomycin (MMC, Jiangsu Henrui Medicine co.,LTD), hydroxy camptothecin (HCPT, Hubei Huangshi Feiyun co., LTD), oxaliplatin (L-OHP, Jiangsu Henrui Medicine co., LTD) and gemcitabine (GEM, Hubei Halfsky pharmacy co., LTD)

\section{Curative effect evaluation}

Curative effect evaluation includes changes in serum tumor marker AFP, tumor size and number, liver function, complications, and overall survival (OS).

\section{Follow-up}

Local therapeutic efficacy was evaluated by contrast-enhanced dynamic CT scanning after 2 courses of chemotherapy or in the case of clinical suspicion for recurrence. Clinical tumor recurrence and response was assessed according to the Response Evaluation Criteria in Solid Tumors (RECIST version 1.0$)^{2}$

\section{Toxicity Assessment}

Toxic effects were assessed during hospitalization of each patient and at the patient baseline according to the National Cancer Institute Common Toxicity Criteria guidelines, version 2.0. CBCs. Liver function status, complications include chemotherapy relative diarrhea and $\mathrm{I}^{0}$ to $\mathrm{II}^{0}$ bone marrow stifled was inspected.

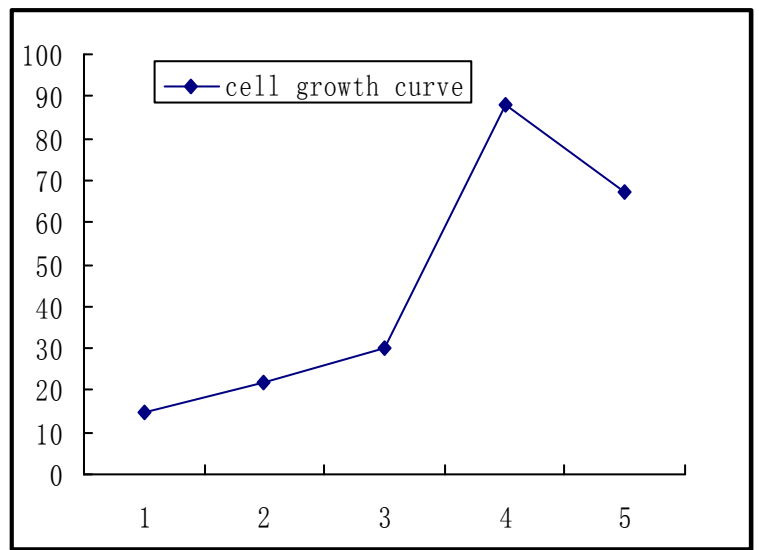

FIG. 1: Growth curve of liver cancer cells cultured in RPMI 1640 (Gibco, Invitrogen) supplemented with $10 \%(\mathrm{v} / \mathrm{v})$ heat inactivated fetal bovine serum and $1 \%(\mathrm{v} / \mathrm{v})$ antimicotic antibiotic at $37^{\circ} \mathrm{C}$ under a $5 \% \mathrm{CO}_{2}$ atmosphere.

\section{Statistical methods}

Clinical responses between two groups were compared by chi-square test. Kaplan-Meier analysis was applied to assess OS, and the significance of differences in survival curves was determined by the log-rank test. OS was defined as the period from the date of chemotherapy to the date of death. $\mathrm{P}<$ 0.05 was considered significant for all analyses. The SPSS software package, version 10.0 (SPSS Inc., Chicago, IL, USA) was used for statistical analysis.

\section{Results}

\section{Cell culture and drug sensitivity test}

Primary tumor cells prepared from the specimens of primary liver tumors obtained by biopsy turned into rapid logarithmic growth phase after cultured for 48 to 72 hours (Figure 1). After 4 days of incubation, chemotherapy drugs were added at the top of the rapid logarithmic phase in the MTT assay. As shown in Figures 2 and 3 and Table 1, different drugs showed different inhibitions to the growth of cancer cells from different patients. In addition, the cells were disrupted and chromatin-mustered when the chemotherapy drugs were added for 24 hours (Figure 4).

TABLE 1: Drug sensitivity test of experimental group

\begin{tabular}{ccc}
\hline \hline Drug & $\begin{array}{c}\text { Tumor suppression } \\
\text { rate }(\%, \mathrm{x} \pm \mathrm{s})\end{array}$ & $\begin{array}{c}\text { Drug-resistant } \\
\text { rate }(\%)\end{array}$ \\
\hline E-ADM & $36.7 \pm 4.5$ & 28.8 \\
HCPT & $33.8 \pm 9.4$ & 31.5 \\
MMC & $29.4 \pm 6.6$ & 33.2 \\
5-FU & $38.5 \pm 7.1$ & 25.4 \\
LOHP & $40.9 \pm 6.3$ & 21.6 \\
DDP & $50.2 \pm 8.8$ & 18.3 \\
GEM & $32.3 \pm 3.9$ & 27.1 \\
\hline \hline
\end{tabular}

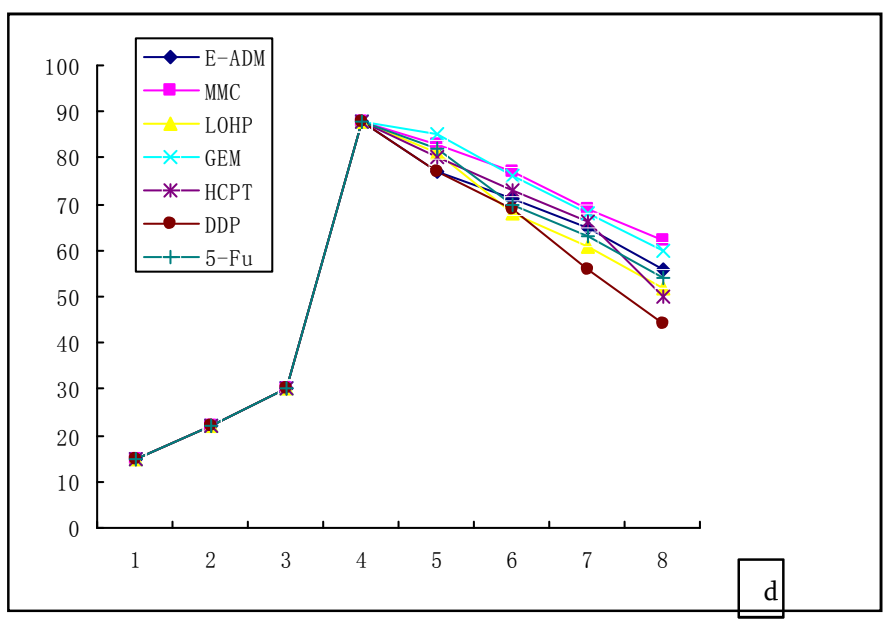

FIG. 2: Inhibition of liver cancer cell growth by chemotherapy drugs added at the top of the logarithmic growth phase. 


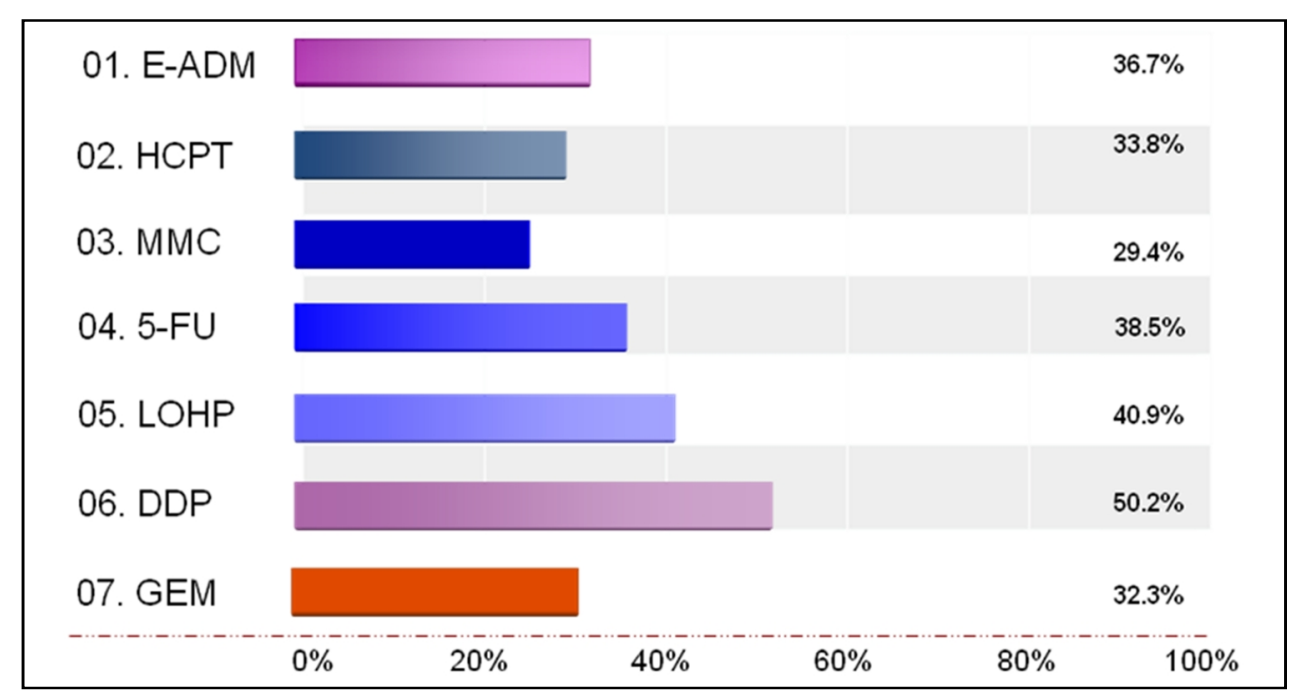

FIG. 3: Different inhibition ratios of 7 anti-cancer drugs to the growth of primary cancer cells. Different drugs have different anti-cancer sensitivities to liver cancer cells.

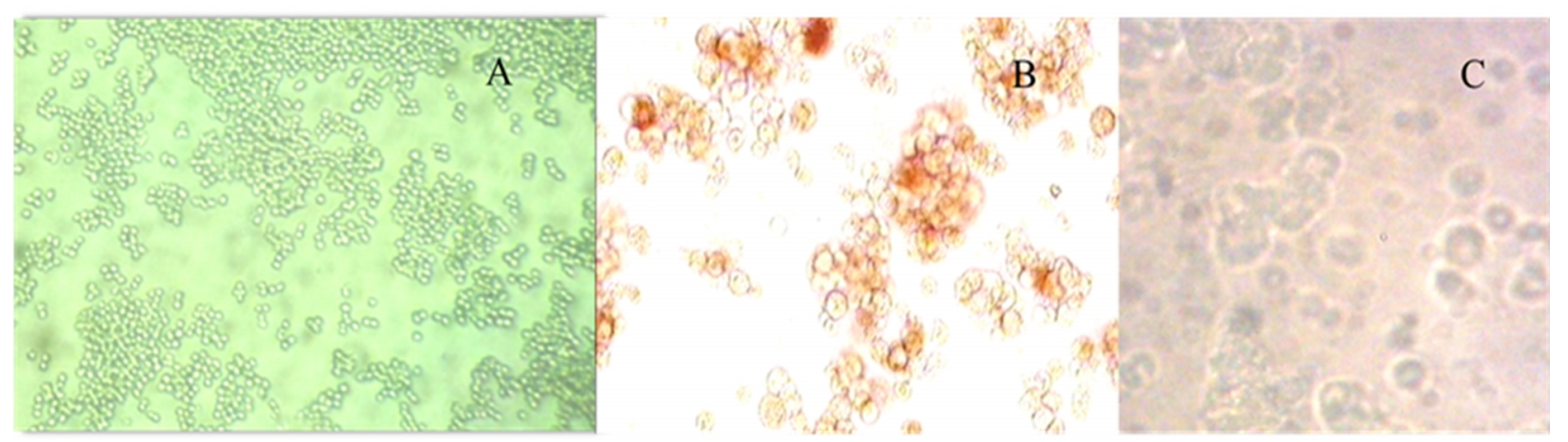

FIG. 4: A. Cultured liver cancer cells began to stick the wall after cultured for 24 hours ( $\times 10)$; B. The cultured liver cancer cells clustered together for 48 hours $(\times 20)$; C. The liver cancer cells were disrupted chromatin-mustered and began to die when the chemotherapy drugs were added for 24 hours.

\section{Clinical observation}

120 cases of unresectable HCC were randomly divided into experimental group and control group, the clinical pathological features of patients in both groups were not significant different (Table 2). The mean patient age at the time of treatment was 56 (range, 47 - 65) years. Bard Access Ports were inserted into the right gastro-omental artery and the right gastro-omental vein to establish regional chemotherapy channels for all patients (Figure 5). Individual patients in the experimental group were infused by arterial and portal vein pump 3 drugs which showed highest inhibition to the cancers of the patient in our MTT test above, whereas the control group patients underwent TACE. In experimental group, the tumor size reduced in $28(47.6 \%)$ cases as measured by CT or MRI after six chemotherapy cycles (Figure 6), stabilized in $14(23.3 \%)$ cases, and progressed in 18 (30\%) cases. The corresponding data was $17(28.5 \%), 7(11.5 \%)$ and 36 $(60 \%)$ cases in the control group $(\mathrm{P}<0.05)$. The AFP was declined in 51 cases in experimental group and in 30 cases in control group $(\mathrm{P}<0.05) .6$ patients in the experimental group and 3 in control group had the opportunity of two-step operation $(\mathrm{P}<0.05)$ (Table 3 ). There was no severe incidence of complications in both groups. In the experimental group, 2 (3\%) cases of patients had wound infection, 8 cases had the chemotherapy relative diarrhea and 18 (30\%) cases had $\mathrm{I}^{0}$ to $\mathrm{II}^{0}$ bone marrow stifled. In the control group, The chemotherapy relative diarrhea was seen in 15 (25\%) cases and $\mathrm{I}^{0}$ to $\mathrm{II}^{0}$ bone marrow stifled was seen in 33 (55\%) cases (Table 4). For prognosis, in the experimental group, the median follow-up time was 21 months, OS rates of 6 months, 12 months, 18 months were $86 \%, 72 \%$, and $65 \%$ respectively. In the control group, the median follow-up time was 16 months, OS rates of 6 months and 12 months were 58\% and $40 \%$, respectively (Figures 7). 
TABLE 2:Patient characteristics: patient and tumor features of the experimental group compared with control group

\begin{tabular}{lccc}
\hline \hline & Experimental group & Control group & P-value \\
$\mathrm{n}$ & 60 & 60 & \\
Age $(\mathrm{Y})$ & & & \\
Median (range) & $56(47-65)$ & $11: 1$ \\
Male : Femal & $53: 7$ & $54(90 \%)$ \\
cirrhosis & $56(93.3 \%)$ & & \\
Primary tumor & & 27 \\
pathologic stage & & 33 \\
II & 21 & \\
III & 39 & $9.3(8.7-13.3)$ \\
Tumor size (cm) & & \\
Median (range) & $9(8-13.7)$ & 37 \\
Tumor number & & 39 \\
1 & 23 & $58(96.7 \%)$ \\
$\geq 2$ & 21 & $1(1.7 \%)$ \\
Hepatitides & & $1(1.7 \%)$ \\
Hepatitis B & $57(95 \%)$ & $0(0 \%)$ \\
Hepatitis C & $1(1.7 \%)$ & $4.18 \pm 2.66$ \\
HepatitisB/C & $0(0 \%)$ & \\
Other & $2(3.3 \%)$ & $40(66.7 \%)$ \\
AFP (Log10ng/ml) & $4.33 \pm 2.52$ & $20(33.3 \%)$ \\
Child-pugh & & $0(0 \%)$ \\
A & $38(63.3 \%)$ & \\
B & $22(36.7 \%)$ & \\
C & $0(0 \%)$ & & \\
Neoplasm & $6(10 \%)$ & \\
Single-tumor & $54(90 \%)$ & \\
Multi-tumor & & \\
\hline \hline
\end{tabular}

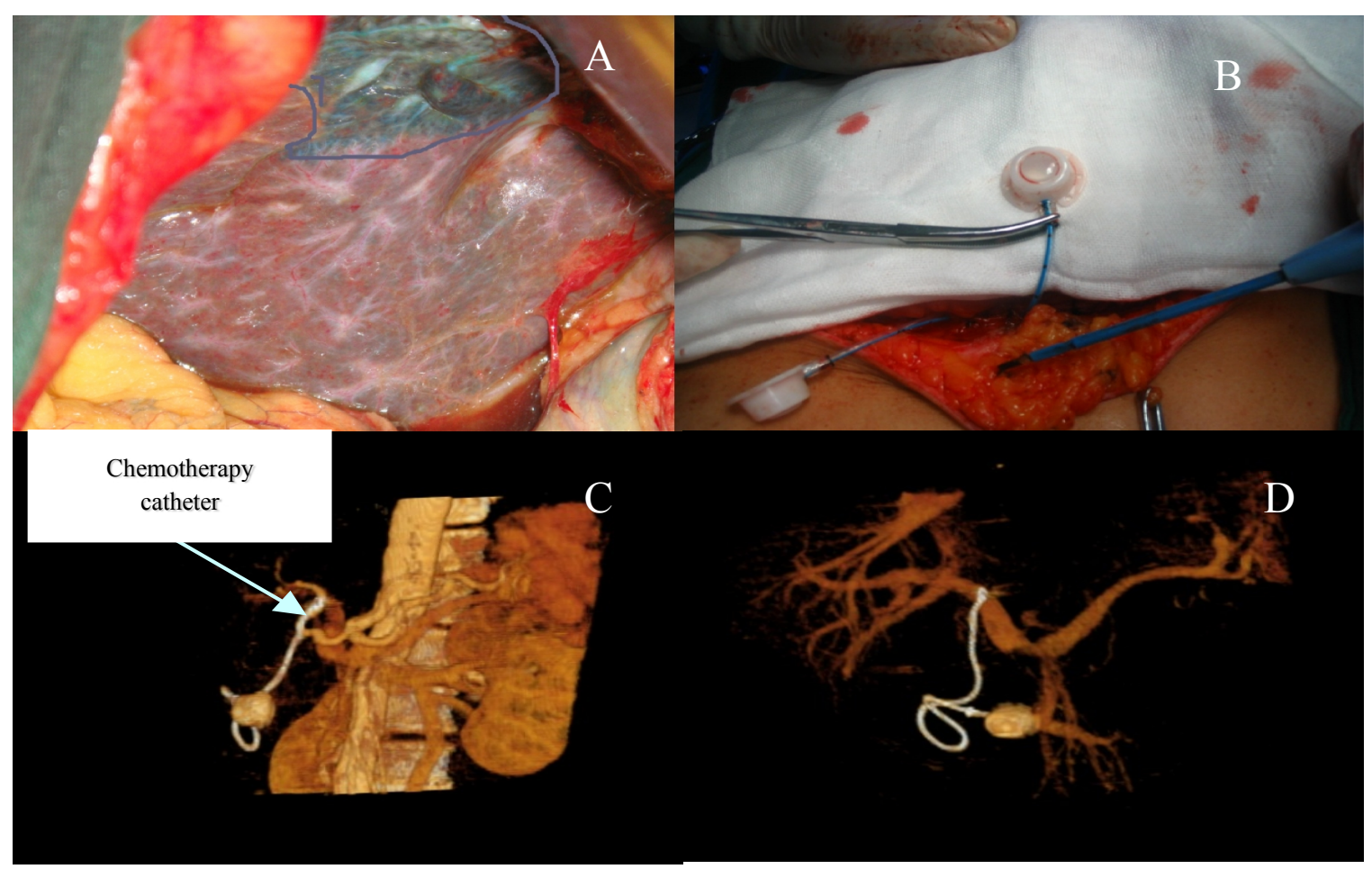

FIG. 5: Bard Access Ports were inserted into the right gastro-omental artery and the right gastro-omental vein to establish regional chemotherapy channels. A. Hepatic artery catheter was inserted into the blood vessel to liver tumor, the liver segment appeared blue after methylene blue staining; B. Operation of insertaion of chemotherapy catheter; C. Artery images showing that the chemotherapy catheter was in the proper hepatica artery; D. Vein imagesshowing that the chemotherapy catheter was in the portal vein. 


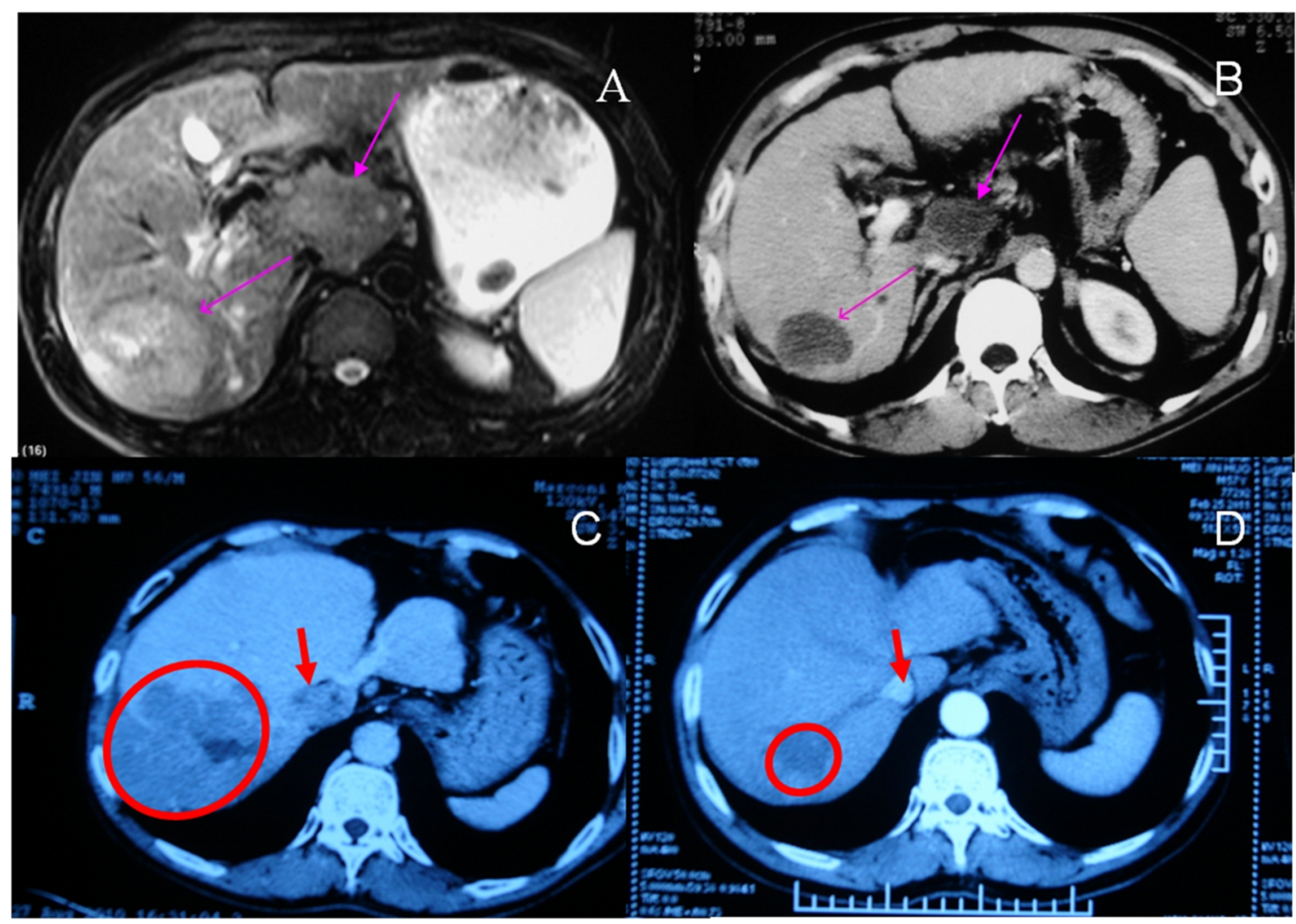

FIG. 6: The tumor size reduced in experimental group as measured by CT or MRI after six chemotherapy cycles. A. MRI showed that the tumor was located in segments I and VII; B. The tumor size reduced and the blood supply decreased obviously after 6 cycles of chemotherapy; C. Tumor located in segment VII and the cells growing into inferior vena cava (IVC) via right hepatic vein; D. The tumor size reduced and the embolus in IVC and right hepatic vein disappeared after 6 cycles of chemotherapy.

\begin{tabular}{lcccccccc}
\multicolumn{1}{l}{ TABLE 3: Clinical effect comparisons between experimental and control groups } \\
\hline \hline Group & $\mathrm{n}$ & \multicolumn{1}{c}{ CT/MRI } & & AFP & Two-step & P-value \\
& & Reduce & stabilize & progress & decrease & rise/ stabilize & operation (n) \\
\hline experimental group & 60 & 28 & 14 & 18 & 51 & 9 & 6 & $<0.05$ \\
control group & 60 & 17 & 7 & 36 & 30 & 30 & 3 & \\
\hline \hline
\end{tabular}

TABLE 4: Complications comparison between experimental and control groups

\begin{tabular}{lcccccc}
\hline \hline Group & $\mathrm{n}$ & wound infection & abdominal pain & hepatic failure & myelosuppression & P-value \\
\hline experimental group & 60 & 2 & 8 & 9 & 18 & $<0.05$ \\
control group & 60 & 0 & 25 & 41 & 33 & \\
\hline \hline
\end{tabular}




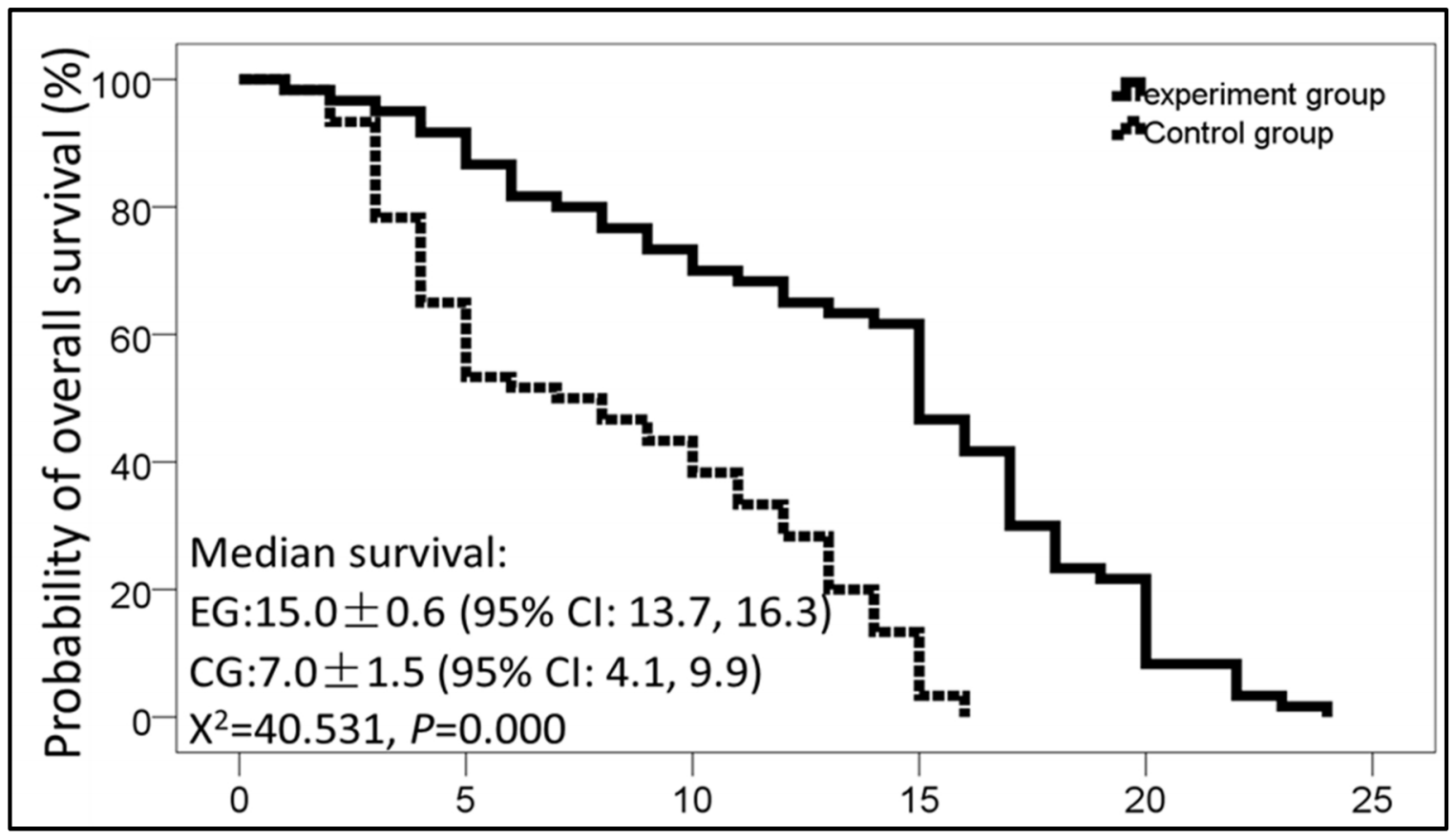

FIG. 7: OS (Overall survival, months) comparison between experimental group and control group

\section{Discussion}

China is a country in which the morbidityof HCC is the highest in the world, and the latest survey data showed that the mortality rate of PHC is the highest in Hubei province. Only less than $20 \%$ of the patients with PHC had the opportunity to be respected. Hepatic trans catheter arterial chemoembolization (TACE) is currently the important therapy of the middle and late stage of $\mathrm{PHC}$ patients with aeffective rate of $30 \% \sim 40 \%$. Research has showed that blood supply to primary liver cancer is through both hepatic artery and portal vein: the tumor center is mainly composed of hepatic artery, and the peripheral part is mainly composed of portal vein. The surrounding part is the most active part of the cancer. ${ }^{3}$ HCC cells invade intrahepatic vascular extremely common, especially the portal vein, taking shape of portal vein embolus and giving rise to intrahepatic metastasis. This is the reason why conventional TACE cannot achieve satisfactory results.

The efficiency of cancer chemotherapy is decided by two factors. The first is drug concentration in the tumor, and the second is the drug effectiveness. Thus, it is important to improve the drug efficiency. In the early 1950's, Bierman first reported hepatic artery perfusion chemotherapy for primary liver cancer. ${ }^{4}$ Storer reported chemotherapy of hepatic neoplasms via umbilical portal vein in $1966^{5}$ and other scholars adopt the hepatic artery or portal vein catheter chemotherapy for the sake of increasing the medicine concentration in tumor, and achieved good efficiency. Here, we used drug sensitivity test to select individualized drugs and perfused the sensitive drugs to individual patients by hepatic artery and portal vein channels, giving consideration to improve the regional drug concentration and tumor susceptibility to drugs and achieve high effectiveness of treatment of PHC.

Surgical resection is the preferred treatment for liver cancer, but because of the low removal rate, the 5 -year survival rate of entire population of liver cancer is less than 5\%.6 Molecular biology research proved that portal venous endothelial cells have specific adhesion molecules which can bind the various components in the blood, including cancer cells, to promote the transfer and spread of tumor cells in the portal vein. ${ }^{78}$ Clinical studies also demonstrated that hepatic artery embolism chemotherapy and portal vein regional chemotherapy of hepatocellular carcinoma after surgical removal can significantly decrease the rate of recurrence and metastasis ${ }^{9}$, and regional chemotherapy through hepatic artery is safe and effective. ${ }^{10}$ Reports for colorectal cancer liver metastasis in patients with arterial perfusion 5 - Fu can obtain curative effect. ${ }^{11}$ With hepatic arterial infusion of fluorouracil plus systemic irinotecan for unresectable hepatic metastases from colorectal cancer, the patients have a response rate of $72 \%$ and the median survival time is 49.8 months. ${ }^{12}$ Case reports also give us encouraging results. For patient with gastric cancer and breast cancer which is metastasized to liver, regional infusion chemotherapy gains curative effect. ${ }^{13,}{ }^{14}$ In neuroendocrine carcinoma liver metastasis, regional infusion chemotherapy is superior to system chemotherapy also. ${ }^{15}$ Primary hepatic carcinoma with III/IV segment portal venous tumor emboli resort to hepatic artery perfusion of cisplatin treatment after resection reached relapse-free survival more than 1 year. ${ }^{16}$ Regional infusion chemotherapy has better prospective than systemic chemotherapy of liver cancer, but there have been no correspond- 
ing treatment standards. ${ }^{17}$ Solid tumors metastatic to the liver had received good prognosis with oxaliplatin, fluorouracil and leucovorin infused from hepatic artery. ${ }^{18}$

For unresectable hepatocellular carcinoma, with continually infusion of low dose fluorouracil and cisplatin by hepatic artery, the liver tumor remission rate was 39\%, and the median survival was 15.9 months. ${ }^{19}$ Tanaka's research showed that hepatic arterial infusion chemotherapy is a safe, effective method to liver cancer patients. ${ }^{20}$ The curative effect is better and side-effect is lower with the chemotherapy catheter infusion..$^{21}$

The sensitivity of tumor cells to chemotherapy drugs can be obtained by drug susceptibility test. ${ }^{22}$ Naitoh et al reported after multicenter clinical trial that culture-drug sensitivity test predicts the outcome of patients undergoing chemotherapy for advanced gastric cancer and can be used as the method to evaluate chemosensitivity. ${ }^{23}$ Taylor found that the survival rate of the group of malignant tumor patients treated with sensitive drugs was obviously higher than that of the drug resistant group and simple surgical treatment group. This research suggested that the drug sensitivity test in vitro guided individualized chemotherapy is effective. ${ }^{24}$

Another question debated is whether the result of the drug susceptibility reaction in vitro is accordance with that in vivo. In vitro results and the in vivo effectiveness may not match exactly ${ }^{25}$, but recent studies have reported that $85.7 \%$ consistency of susceptibility test in vitro and clinical observation. ${ }^{26}$ Kabeshima and Taylor's research showed that the patients' long-term survival rate was obviously higher in drug-sensitive group than that of drug resistant group and simple surgical group, this indicates that drug sensitivity test in vitro can play an important role in directing the individualized chemotherapy and improving the curative effect. ${ }^{24,27}$ Thus, we carried out this study and our result is encouraging.

Compared with systemic chemotherapy, regional chemotherapy can have these advantages: (1) chemotherapy drug concentration in the tumor increases significantly and makes chemotherapy drug acts on the tumor tissues directly and efficiency. (2) Along with the increase of the drug concentration in the tumor, curative effect increase accordingly. However, it is a critical factor of finding effective chemotherapy drugs for the patients and administrates these drugs by regional catheters.

Seven anticancer drugs were chosen in our study for in vitro drug sensitivity test in 60 cases of patients with unresectable HCC. The results showed that the tumor inhibition rate is different in seven anticancer drugs, and different drug have different resistance rate. Chemotherapy guided by drug susceptibility test shows that the tumor response rates, including the changes of AFP, OS, and second-stage-resection, were superior in experimental group than that of control group, whereas the side effects were relatively small and the survival rate was higher.
In the dual channel infusion chemotherapy under the guidance of drug susceptibility test, regional chemotherapy has better clinical benefit than systemic chemotherapy, providing us a useful way for patients' treatment with primary liver cancer. However, because there were cases in which we failed to detect sensitive chemotherapy drugs in the experimental group, more drugs should be tested in future studies.

\section{Conclusion}

The artery and portal vein pump transfusion chemotherapy guided by drug sensitive test was efficient for HCC treatment. The patients can get longer OS and lower complication incidence.

\section{Acknowledgement}

This study was supported by the Outstanding Medical Academic Leader Projection (2013) in Hubei, China.

\section{Conflict of interest}

The authors declare that they have no conflicts of interest. The authors alone are responsible for the content and writing of the paper.

\section{References}

1. Shiina S, Teratani T, Obi S, et al. Nonsurgical treatment of hepatocellular carcinoma: from percutaneous ethanol injection therapy and percutaneous microwave coagulation therapy to radiofrequency ablation. Oncology 2002; 62:64-8.

2. Therasse P, Arbuck SG, Eisenhauer EA, et al. New guidelines to evaluate the response to treatment in solid tumors. European Organization for Research and Treatment of Cancer, National Cancer Institute of the United States, National Cancer Institute of Canada. JNatl Cancer Inst 2000; 92:205-16.

3. Minami Y, Kudo M. Hepatic malignancies:Correlation between sonographic fingings and pathological features. World J Radiol. Jul 28, 2010; 2: 249-256.

4. Bierman HR, Byron RL Jr, Miller ER, Shimkin MB. Effects of intra-arterial administration of nitrogen mustard. Am JMed 1950; 8:535.

5. Storer E, Akin T. Chemotherapy of hepatic neoplasms via the umbilical-portal vein. Am J Surg 1966; 111:56-65.

6. Katanoda K, Matsuda T. Five-year relative survival rate of liver cancer in the USA,Europe and Japan. Jap J Clin Oncol 2014; 44: 302-3.

7. Liu LX, Jiang HC, Liu ZH, et al. Integrin gene expression profiles of human hepatocellular carcinoma. World J Gastroenterol 2002; 8:631-7. 
8. $\mathrm{Ng} \mathrm{IO,} \mathrm{Liu} \mathrm{CL,} \mathrm{Fan} \mathrm{ST,} \mathrm{Ng} \mathrm{M.} \mathrm{Expression} \mathrm{of}$ $\mathrm{P}$-glycoprotein in hepatocellular carcinoma. A determinant of chemotherapy response. Am J Clin Pathol. 2000; 113:355-63.

9. Tung-Ping Poon R, Fan ST, Wong J. Risk factors, prevention, and management of postoperative recurrence after resection of hepatocellular carcinoma. An Surg 2000; 232:10-24.

10. Ueda H, Fukuchi H, Tanaka C. Toxicity and efficacy of hepatic arterial infusion chemotherapy for advanced hepatocellular carcinoma (Review). Oncol Lett 2012; 3:259-63.

11. Melichar B, Voboril Z, Krajina A, et al. Hepatic arterial infusion of irinotecan, 5-Fluorouracil and leucovorin in patients with liver metastases from colorectal carcinoma. Anticancer Res 2012; 32:5487-93.

12. Arai Y, Ohtsu A, Sato Y, et al. Phase I/II study of radiologic hepatic arterial infusion of fluorouracil plus systemic irinotecan for unresectable hepatic metastases from colorectal cancer: Japan Clinical Oncology Group Trial 0208-DI. J Vasc Interv Radiol 2012; 23:1261-7.

13. Yamamoto Y, Maruyama T, Tanaka H, et al. A case of a patient with gastric cancer and liver metastasis -a long-term survival of 10 years. Gan To Kagaku Ryoho 2012; 39:2375-7.

14. Satoh E, Ebuchi M, Maruyama M, et al. A case report of liver metastasis of breast cancer successfully treated by hepatic arterial infusion of docetaxel and systemic administration of nab-paclitaxel. Gan To Kagaku Ryoho 2012; 39:2066-7.

15. Mayo SC, Herman JM, Cosgrove D, et al. Emerging approaches in the management of patients with neuroendocrine liver metastasis: role of liver-directed and systemic therapies. J Am Coll Surg 2013; 216:123-34.

16. Mukai Y, Takeda Y, Nakahira S, et al. A case of successful treatment with intra-arterial infusion chemotherapy with cisplatin for advanced hepatocellular carcinoma with portal vein tumor thrombus. Gan To Kagaku Ryoho 2012; 39:1815-8.

17. Tsochatzis EA, Fatourou EM, Triantos CK, Burroughs AK. Transarterial therapies for hepatocellular carcinoma. Recent Results Cancer Res 2013; 190:195-206.
18. Tsimberidou AM, Leick MB, Lim J, et al. Dose-finding study of hepatic arterial infusion of oxaliplatin-based treatment in patients with advanced solid tumors metastatic to the liver. Cancer Chemother Pharmacol 2013; 71:389-97.

19. Ueshima K, Kudo M, Takita M, Nagai T, Tatsumi C, Ueda T, Kitai S, Ishikawa E, Yada N, Inoue T, Hagiwara S, Minami Y, Chung H. Hepatic arterial infusion chemotherapy using low-dose 5-fluorouracil and cisplatin for advanced hepatocellular carcinoma. Oncology. 2010; 78 Suppl 1: 148-53.

20. Tanaka M, Fukumoto T, Kido M, et al. Analysis of the safety and efficacy of percutaneous isolated hepatic perfusion after particletherapy for advanced hepatocellular carcinoma. Gan To Kagaku Ryoho2013 Nov; 40:1681-3.

21. Ueda H, Fukuchi H, Tanaka C. Toxicity and efficacy of hepatic arterial infusion chemotherapy for advanced hepatocellular carcinoma (Review). Oncol Lett 2012; 3:259-63.

22. Shimizu D, Ishikawa T, Ichikawa Y, et al. Current progress in the prediction of chemosensitivity for breast cancer. Breast Cancer 2004; 11:42-8.

23. Naitoh H, Yamamoto H, Murata S, Kobayashi H, Inoue K, Tani T. Stratified phase II trial to establish the usefulness of the collagen gel droplet embedded culture-drug sensitivity test (CD-DST) for advanced gastric cancer. Gastric Cancer. 2013. [Epub ahead of print]

24. Taylor CG, Sargent JM, Elgie AW, et al. Chemosensitivity testing predicts survival in ovarian cancer. Eur J Gynaecol Oncol 2001; 22:278-82.

25. Schrag D, Garewal HS, Burstein HJ, et al. American Society of Clinical Oncology Technology Assessment: chemotherapy sensitivity and resistance assays. JClin Oncol 2004; 22:3631-8.

26. Arienti C, Tesei A, Verdecchia GM, et al. Peritoneal carcinomatosis from ovarian cancer: chemosensitivity test and tissue markers as predictors of response to chemotherapy. J Transl Med 2011; 9: 94-101.

27. Kabeshima Y, Kubota T, Watanabe M, et al. Clinical usefulness of chemosensitivity test for advanced colorectal cancer. Anticancer Res 2002; 22:3033-7. 